\title{
A Calcified Artery of Drummond, Could It Be a Sentinel Sign of Further Complications?
}

\author{
Shahe Boghossian, Arpan K. Banerjee \\ Heart of England NHS Trust, Birmingham, UK \\ Email: shaboghru@gmail.com
}

Received March 24, 2012; revised April 30, 2012; accepted May 18, 2012

\begin{abstract}
Objective: To understand the role of the artery of Drummond in the elderly population. The artery plays a crucial role in the anastomosis between the inferior mesenteric artery and superior mesenteric artery. Its maintenance is particularly important for the survivability of the bowel in events of stenosis of either one of the native arteries; SMA or IMA. Method: A 94 year old lady presents with post-prandial abdominal pain and significant emaciation. The patient undergoes clinical and radiological investigations to find out the cause of her ailments. Results: Abdominal X-ray revealed a serpentine structure that was later defined as the artery of Drummond, CT scan revealed a calcified and enlarged artery of Drummond in addition to an occluded origin of SMA and celiac artery as well as a severe stenosis origin of IMA. Conclusion: The artery of Drummond was immensely hypertrophied and was supplying the entire bowel through the native IMA; subsequent occlusion of the IMA was the main cause that caused the demise of the patient.
\end{abstract}

Keywords: Artery of Drummond; Mesenteric Ischemia; Refeeding Syndrome

\section{Case Report}

A 94 year old lady was admitted to Accident and Emergency complaining of six week history of abdominal pain, nausea and significant emaciation. She was alert with a GCS of 15. Examination of the abdomen revealed two well healed midline incisions. Palpation of the abdomen revealed fullness on the left side of her abdomen as well as a hard palpable tube like structure that rolled underneath the examiner's fingers. Auscultation of her abdomen showed faint yet audible bowel sounds. Rectal examination showed an empty rectum. Chest X-ray showed bibasal collapse but no obstruction of the bronchi and no signs of any pulmonary malignancy.

On admission, her initial blood test showed the following: Na: 132, K: 4.2, Creatinine: $82 \mathrm{mmol} / \mathrm{lit}$, Alb: 40, Bilirubin: 16, Phosphate: 1.02, Hemoglobin: 14.4, Platelets: 252, White cell count: 11.32. Her observation chart was normal however the patient looked dehydrated and hence the patient was put on a slow IV drip. The patient looked generally emaciated and was put on IV rehydration, oral rehydration, as well as fortified oral protein sachets. The patient was not able to tolerate the oral feed and hence a provisional diagnosis of Refeeding Syndrome was put forward. Three days later, a random arterial blood gas showed an elevation of serum lactate to 6.5 $\mathrm{mmol} / \mathrm{lit}$ with a base excess of $-3.0 \mathrm{mmol} / \mathrm{lit}, \mathrm{pCO}_{2}: 4.58$ $\mathrm{kPa}, \mathrm{pO}_{2}: 12.0 \mathrm{kPa}, \mathrm{pH}: 7.4$ and a CRP of 161 . Her white cell count was elevated at 19.38/ml. Unfortunately, the patient expired a week later.

\section{Findings}

The abdominal and chest X-ray showed a calcified transversely oriented tube like structure the crossed the full spectrum of the abdomen (Figure 1). This was initially thought to be a foreign body but later confirmed to be a calcified vascular structure. Contrast enhanced Computer Tomography confirmed the structure to be a calcified artery of Drummond (Figure 2) with a stenosed origin of the Celiac, Superior Mesenteric Artery (Figure 3) and the Inferior Mesenteric Artery (Figure 4). The diagnosis eventually confirmed mesenteric ischemia in the elderly.

\section{Discussion}

Chronic mesenteric ischemia is an indolent condition affecting mainly the vascular supply to the small bowel. It is mainly manifested by postprandial pain and subsequent weight loss. The most common artery involved is the Superior Mesenteric Artery (SMA) which supplies the duodenum, the jejunum, the ileum as well as the right side of the large bowel [1]. Occasionally, the coeliac artery may be involved as well, either as a solitary condition or in association with widespread atherosclerosis of the Aorta. Congenital conditions such as acute angulation of the SMA with the Aorta or fibromuscular dysplasia have been 


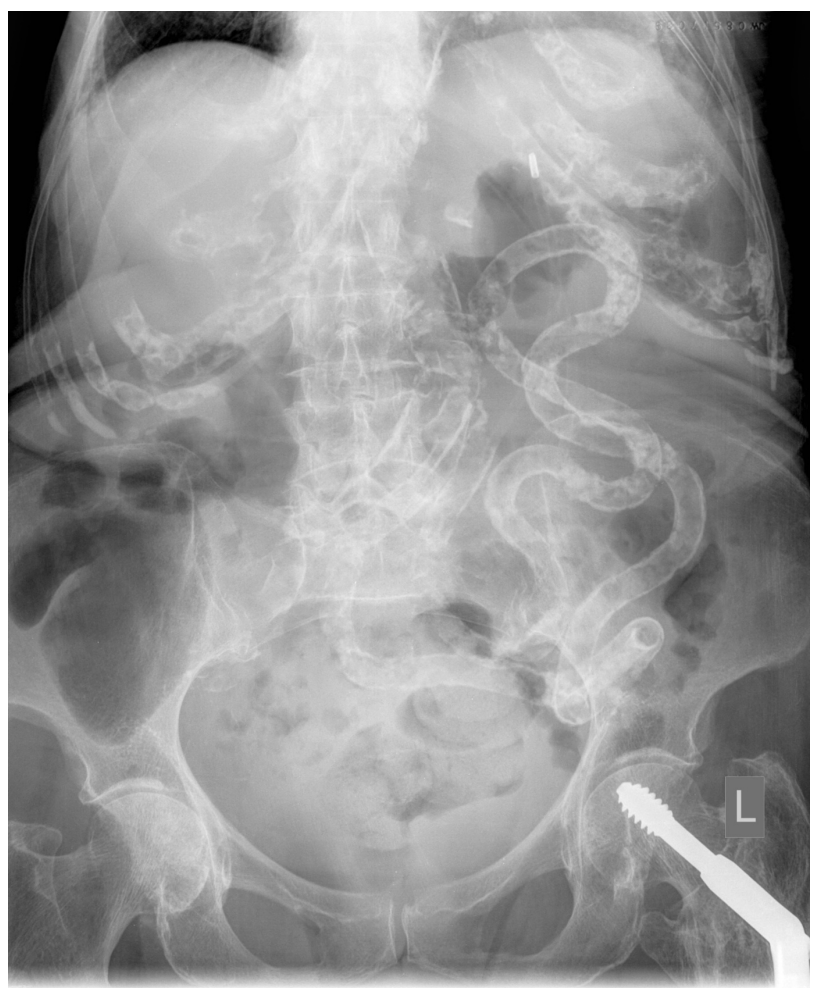

Figure 1. Showing a very calcified tubular structure that was initially confused as a foreign body.

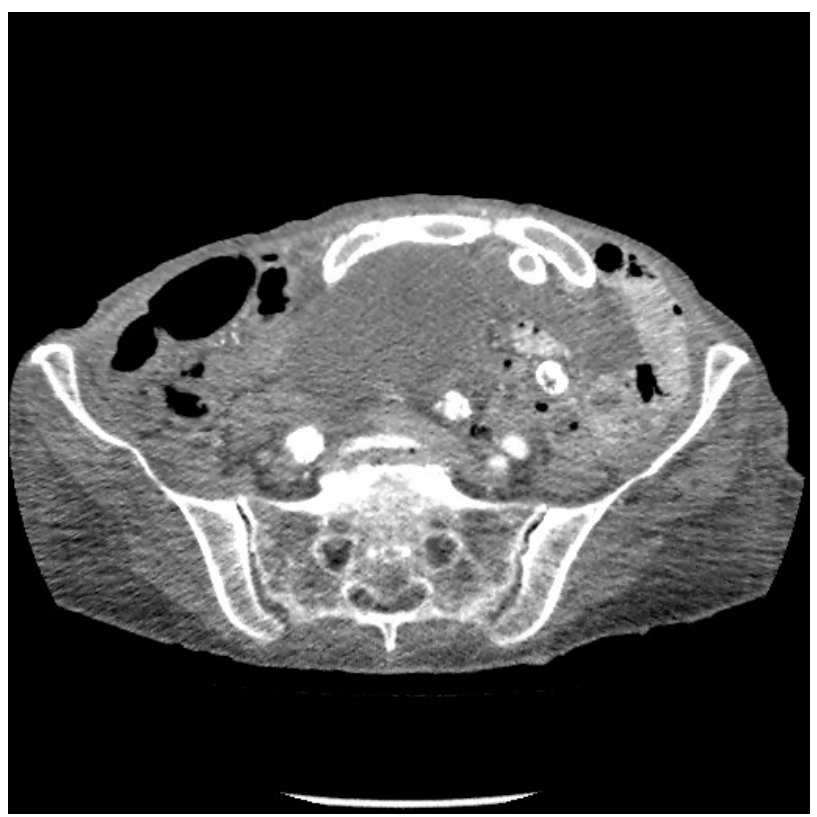

Figure 2. Showing a calcified tubular structure at the very ventral edge of the patient's abdomen.

sporadically reported [2]. Acquired yet non-occlusive mesenteric ischemic (NOMI) conditions include atherosclerotic degeneration of the origin of the SMA, vasculitides affecting the SMA as well as other mesenteric vessels, thrombosis of the left renal vein causing indirect

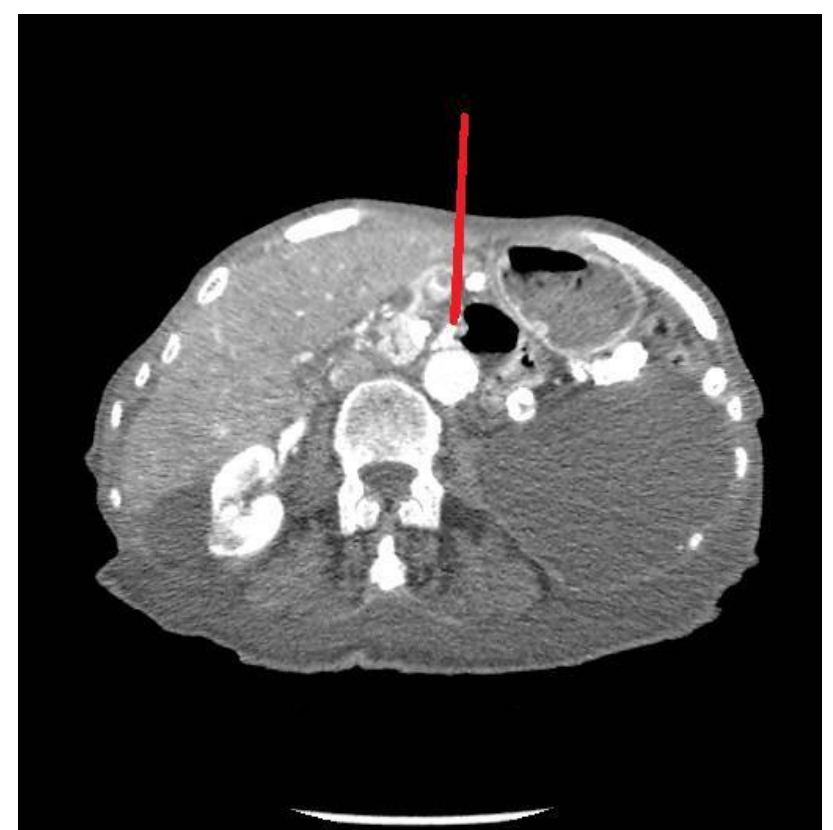

Figure 3. Shows no flow from the orifice of the SMA (red pointer) and a calcified meandering structure on the surface of the skin.

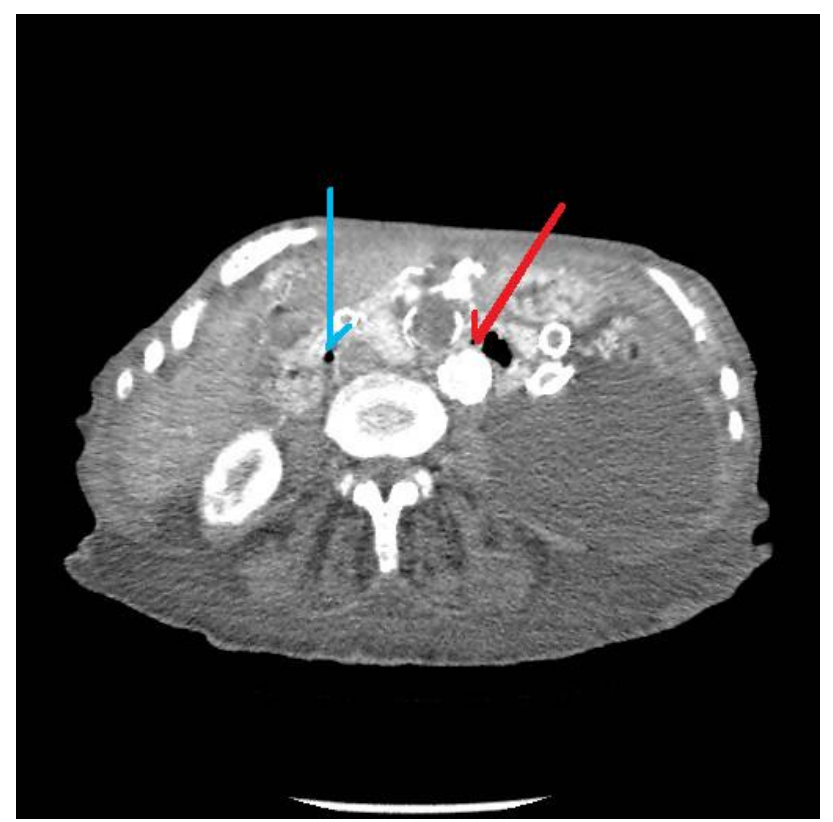

Figure 4. Shows no flow in the IMA (red pointer) and already shows a miniscule amount of gas in the luminal bowel (blue pointer).

obstruction to the origin of the SMA, paraaortic tumours and lymphadenopathy, pancreatitis [3] and hypercoagulable states [4,5]. In contrast, the most common cause of acute mesenteric ischemia is mainly atrial fibrillation, hypercoagulable states as well as septic emboli from the cardiac valves or other sources in the body [6]. In the elderly, as discussed earlier, background atherosclerotic 
disease is a common finding on non-invasive imaging studies, as demonstrated by the images shown underneath (CT and AXR). However, in an acute ischemic event, the anastamotic feeder vessel would thrombose rendering the bowel non-viable and causing irreversible ischemic injury. This case highlights a variant whereby the coeliac axis territory and the SMA territory are supplied by a dominant artery of Drummond which in turn is supplied by the IMA as demonstrated by abdominal imaging. The artery of Drummond is a crucial anastomosis between the superior and inferior mesenteric artery. Additionally the viability of this arc is crucial for the survivability of Endovascular Aortic Aneurysm repairs whereby the origin of the inferior mesenteric artery is iatrogenically occluded $[7,8]$. Our assessment of the patient's condition certainly demonstrated that she had long standing atherosclerotic disease as described from her weight loss, but we can certainly say that acute embolisation of the inferior mesenteric artery (IMA) was a key factor in her rapid deterioration. The case highlights a case of hypertrophy of the artery of Drummond that was crucial in maintaining nutrient supply to the entire foregut and hindgut through the IMA.

\section{REFERENCES}

[1] P. R. Kvietys, “The Gastrointestinal Circulation,” Morgan \& Claypool Life Sciences, San Rafael, 2010.

[2] R. M. Hamed, and K. Ghandour, “Abdominal Angina and
Intestinal Gangrene-A Catastrophic Presentation of Arterial Fibromuscular Dysplasia: Case Report and Review of the Literature,” Journal of Pediatric Surgery, Vol. 32, No. 9, 1997, pp. 1379-1380. doi:10.1016/S0022-3468(97)90328-6

[3] M. Hirota, K. Inoue, Y. Kimura, et al., "Non-Occlusive Mesenteric Ischemia and Its Associated Intestinal Gangrene in Acute Pancreatitis,” Pancreatology, Vol. 3, No. 4, 2003, pp. 316-322. doi:10.1159/000071770

[4] N. Scally, M. E. O’Donnell, C. McClintock, M. Allen, S. J. Kirk and S. Dolan, "Mesenteric Artery Occlusion Secondary to Activated Protein C Resistance: A LifeThreatening Combination," Angiology, Vol. 58, No. 6, 2008, pp. 768-771.

[5] N. Agaoglu, S. Turkyilmaz, C. Ozlu, M. K. Arslan and E. Colak, "Significance of Antithrombin III, Protein C and Protein S in Acute Mesenteric Ischemia Patients," Acta Chirurgica Belgica, Vol. 104, No. 2, 2004, pp. 184-186.

[6] D. Hussain, S. L. Sarfraz, S. K. Baliga and R. Hartung, "Acute Mesenteric Ischemia: Experience in a Tertiary Care Hospital,” Journal of Ayub Medical College Abbottabad, Vol. 21, No. 4, 2009, pp. 70-72.

[7] J. Kulhanek, R. Chiu, S. Wann, "Multislice CT Angiographic Reconstruction of Marginal Artery of Drummond as Collateral to Occluded Superior Mesenteric Artery," American Heart Hospital Journal, Vol. 4, No. 4, 2006, pp. 290-291. doi:10.1111/j.1541-9215.2006.04931.x

[8] D. L. Bhatt, "Guide to Peripheral and Cerebrovascular Intervention,” Remedica, London, 2004. 